\title{
Primary Paraspinal Malignant Peripheral Nerve Sheath Tumor
}

\author{
Kyoung-Tae Kim, M.D., Yong-Suk Park, M.D., Jeong-Taik Kwon, M.D., Young-Baeg Kim, M.D. \\ Department of Neurosurgery, College of Medicine, Chung-Ang University, Seoul, Korea
}

\begin{abstract}
Malignant peripheral nerve sheath tumors (MPNSTs) are very rare tumors. We experienced a case of MPNST in the cervical paraspinal space which was not associated with neurofibromatosis. The tumor located in left C6-7 foramen and compressed C7 root. The tumor was removed through the occipital triangle. We report a case of the primary cerivcal MPNST in a patient who did not have neurofibromatosis-1.
\end{abstract}

KEY WORDS : Malignant peripheral nerve sheath tumor · Cervical paraspinal space · Occipital triangle.

\section{INTRODUCTION}

Malignant peripheral nerve sheath tumors (MPNSTs) are very rare tumors. The tumor used to be called by various names, including malignant schwannoma, neurofibrosarcoma, neurogenic sarcoma, and malignant neurilemmoma ${ }^{13)}$. Neurofibromatosis 1 (NF1) is the most important risk factor, as approximately $50-60 \%$ of MPNSTs occur in patients with NF1. The incidence of MPNST among patients with NF1 is approximately $10 \%{ }^{8)}$. MPNSTs prefer to locate in the extremities and most of paraspinal MPNST is metastatic lesion, so primary cervical MPNST is very rare $^{6,9)}$. We report a case of the primary cervical MPNST with review of the literatures.

\section{CASE REPORT}

A 56-year-old male presented with the left arm radiating pain (C7 dermatome), which developed one year ago. He was treated with conservative treatment in the primary hospital for 2 months, but his symptom did not improve. On neurological examination, the motor power was normal, but hypoesthesia developed on the C7 dermatome of the left arm.

Computerized tomography (CT) showed a $2 \times 4 \mathrm{~cm}$

- Received : April 1, 2008 •Accepted : July 24, 2008

- Address for reprints : Young-Baeg Kim, M.D.

Department of Neurosurgery, College of Medicine, Chung-Ang

Unveristy, 224-1, Heukseok-dong, Dongjak-gu, Seoul 156-755, Korea

Tel : +82-2-6299-1598, Fax : +82-2-821-8409

E-mail : ybkim1218@cau.ac.kr sized mass lesion in left paraspinal space. The mass was iso-dense and mildly enhanced around periphery (Fig. 1). Magnetic resonance image (MRI) showed a $2 \times 4 \mathrm{~cm}$ sized lobulating mass in left paraspinal space. This lesion was growing into left neural foramen at C6-7 level and showed well-enhancement and multiple internal necrotic change (Fig. 2). The mass compressed a C7 nerve root. Preoperative electromyography (EMG) revealed left-sided C6-7 radiculopathy. There were no evidence of the metastasis on the Positron Emission Tomography (PET)-CT and high resolution CT (HRCT).

The patient underwent surgery using a posterior approach. The tumor was completely removed as one piece through the occipital triangle. On operative field, the tumor margin was relatively clear without any specific attached site. We confirmed the intact $\mathrm{C} 7$ root after the tumor removal and surgical margins were checked by frozen biopsy.

A pathologic examination, which included various imm-
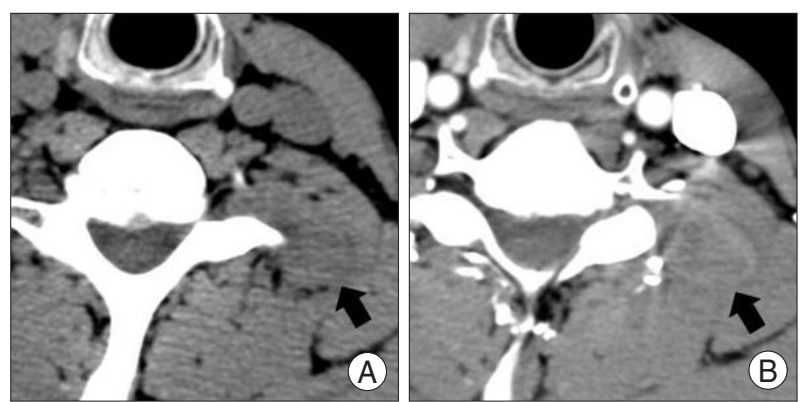

Fig. 1. Computerized tomography showing a $2 \times 4 \mathrm{~cm}$ sized mass lesion in left paraspinal space (black arrow). The mass is iso-density and mild rim-enhanced. A : Pre-enhanced, B : Post-enhanced. 

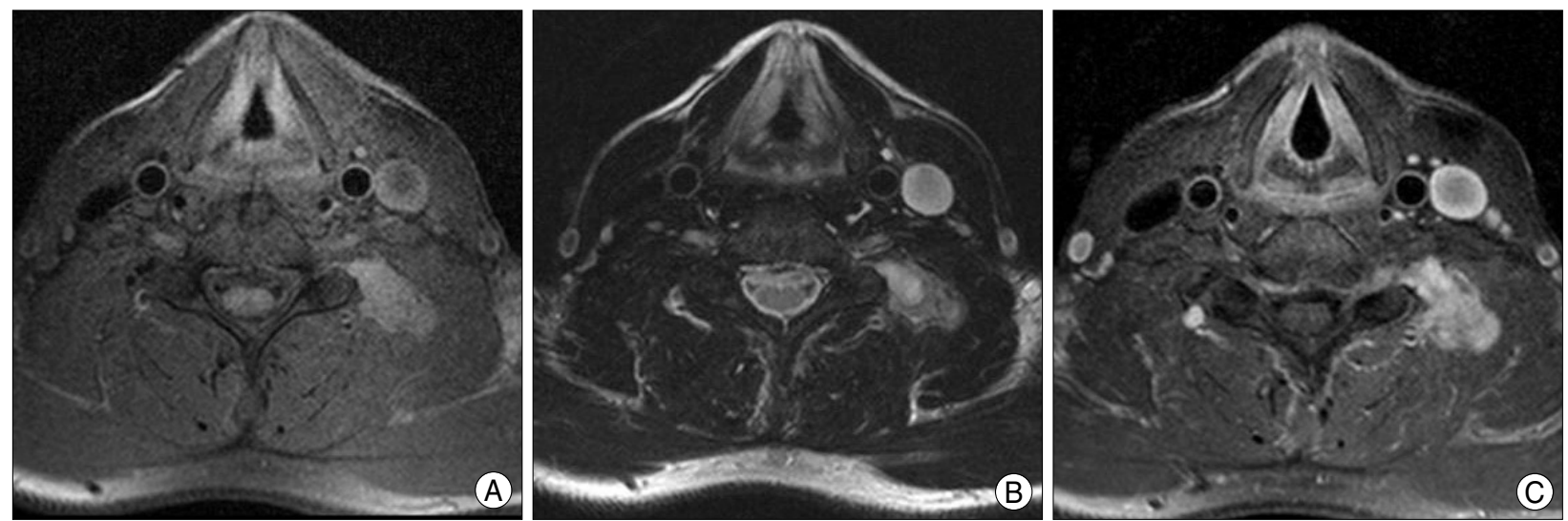

Fig. 2. Magnetic resonance image showing a $2 \times 4 \mathrm{~cm}$ sized lobulating mass lesion in left paraspinal space. This lesion is growing into left neural foramen at C6-7 level and shows well-enhancement and multiple internal necrotic change. A : T1WI. B : T2WI. C : T1 enhanced.

unostainings, demonstrated spindled cells arranged in a fascicular growth pattern and well-differentiated epithelioid areas with necrosis, with irregularly shaped cells characteristic of Schwann cells. The tumor cells had abundant cytoplasms and round to oval nuclei with a distinct cytoplasmic borders. On the immunohistochemical staining, some portions of the tumor were strongly positive for S-100 protein, but negative for CD34, EMA (Endothelial membrane antigen) and GFAP (Glial fibrillary acid protein). The histopathological findings of the mass proved to be a
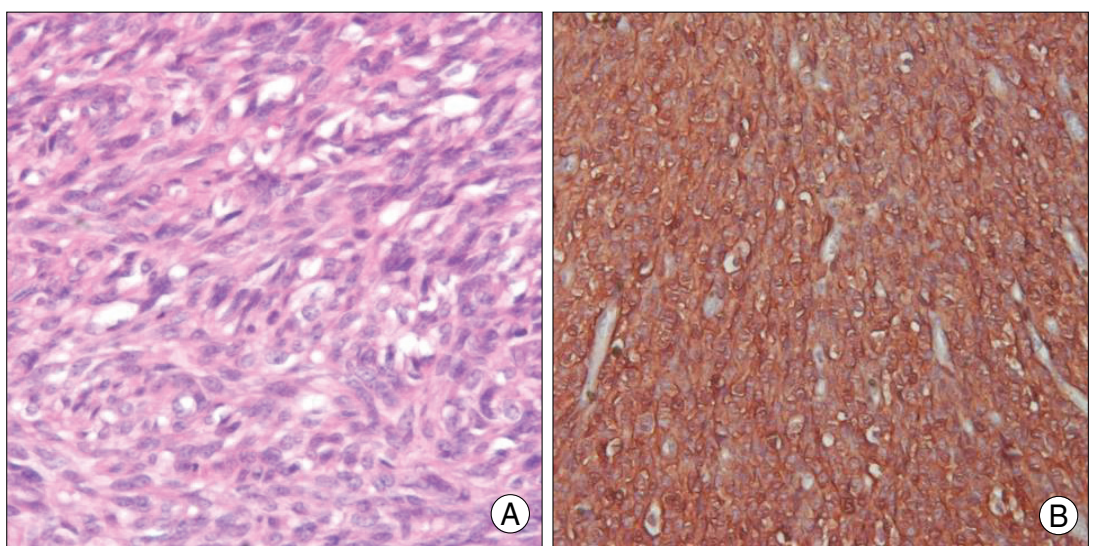

Fig. 3. On microscopic finding, the tumor has two cellular portions, which are composed of crowded spindled cells with hyperchromatic nuclei (upper portion) and epithelioid cells with abundant cytoplasm and prominent nucleoli (lower portion) (hematoxylin and eosin stain, $\times 400)(A)$. Epithelioid portion of the tumor typically shows strong immunoreactivity to $\mathrm{S}-100$ protein (B).

mixed type MPNST (Fig. 3).

The postoperative course was uneventful without any complication. The pain was relieved and the patient was referred to the radio-oncologic center for the radiation therapy. He was treated with intensity-modulated radiation therapy (IMRT) for 2 months and showed no evidence of local recurrence and metastasis on PET CT and neck MRI performed 6 months after the operation.

\section{DISCUSSION}

Malignant peripheral nerve sheath tumors (MPNSTs) are rare tumors with a reported incidence of 1 per $10^{6}$ people per year ${ }^{15)}$. In particular, primary cervical MPNST was very rare tumor. Approximately 50-60\% of MPNSTs occur in patients with $\mathrm{NF}^{8}{ }^{8}$. Most of MPNST occurs in the deep soft tissue, usually close to a nerve trunk. The most common sites are the sciatic nerve, brachial plexus, and sacral plexus ${ }^{9,14)}$.

The diagnosis of MPNST is very difficult, so evaluation should begin with a complete history and physical exam- ination, if MPNST was suspected. Medical history should include a neurogenic disorder (e.g., NF1) or family history of neurogenic disorders. Physical examination should include a skin and neurologic evaluation. Needle biopsy of suspected tumor is the most popular diagnostic tool ${ }^{16}$. The radiologic findings of MPNST are various. At CT, the density of MPNST depends on their histologic characteristics. CT scanning may also be useful in the primary evaluation of patients with MPNST (Fig. 1). CT provides fine features on the relationship of the tumor to surrounding important structures. However, there are no specific CT findings which distinguish MPNST from neurofibroma ${ }^{4}$. Also, MRI has limited utility in distinguishing MPNSTs from benign peripheral nerve lesions. The 'target sign', a central hypointense region seen on T2 weighted images, may be helpful in diagnosing benign neurofibroma. By contrast, MPNSTs classically lack the target sign. Findings on MRI that are suggestive of malignancy include : increase in the size of previously stable neurofibromas, large size, inhomogeneity, ill-defined margins, invasion of fat planes, 
and perilesional edema ${ }^{9,12,14,15)}$. MRI findings of our case, in which the tumor was hyperintense on T1, T2WI and well enhanced, suggested MPNST.

The majority of MPNST are composed of spindled cells arranged in a fascicular growth pattern similar to a fibrosarcoma, but fewer than 5\% of MPNSTs are either partially or purely epithelioid ${ }^{10,15)}$. At the low end of the grading spectrum (WHO grade II), MPNSTs merge with cellular neurofibromas, from which they are distinguished on the basis of increased cellularity, nuclear size ( $>3 \mathrm{x}$ that of neurofibroma cells) and hyperchromasia (Fig. 3A) ${ }^{11)}$. Also, epithelioid MPNST characterizes by increased cellularity, abundant cytoplasm and prominent nuceloli (Fig. 3A). S-100 protein is the most commonly used antibody to identify various nerve sheath tumors, but only $50-70 \%$ of MPNSTs exhibit S-100 protein staining ${ }^{11,15,18)}$. Reactivity is grade-related. In high-grade tumors it is either patchy or found in individual cells, whereas in low-grade examples it may be extensive ${ }^{11,15,18)}$. Our case showed strong immunoreactivity to S-100 protein (Fig. 3B), as with mixed type MPNST, which have a well differentiated epithelioid portion.

The treatment of MPNSTs represent a challenge. Surgical resection is the first line of therapy. The goal of operation is complete removal of the tumor with histologically clear margins of resection. This resection often requires en-bloc resection of major nerves and acceptance of potentially significant functional $\operatorname{loss}^{8,16}$. Resectability rates are related to tumor location and range from 20 (paraspinal) to $95 \%$ $(\text { extremity })^{2)}$. In our case, the tumor located in the adjacent area of C6-7 foramen. Fortunately, the tumor was completely removed through the occipital triangle, composed of a sternocleidomastoid muscle (anterior border), trapezius muscle (posterior border) and omohyoid muscle (inferior border), without functional loss.

MPNSTs have been demonstrated to have a higher local recurrence rate compared to other soft tissue sarcomas ${ }^{10}$. The effect of the postoperative adjuvant therapy for MPNST is not clear. Moreover, the role of chemotherapy in the treatment of MPNSTs tumors has never been adequately studied and remains controversial. However, the effect of radiation therapy has been proved by various studies ${ }^{5,10,13,16)}$. Carli et al. have reported improved local control in MPNST patients treated with radiation therapy ${ }^{5}$. This makes the use of radiation therapy conceptually attractive in patients with MPNST. Hematogenous metastatic spread occurs most commonly to the lungs. Therefore, high resolution chest CT (HRCT) should be performed on all patients with MPNSTs to evaluate for pulmonary metastases $^{3)}$.
Despite aggressive surgery and adjuvant therapy, the prognosis remains poor with a 5-year survival ranging from $16 \%$ to $53 \% \%^{1,7,9,17,19)}$. The 5 -year local recurrence and distant metastasis rates have been previously reported to be $27-42 \%$ and $26-65 \%$ respectively ${ }^{1,67,7,16,17,19)}$. A number of different prognostic factors have been cited in published series as follows: 1) the site, 2) the tumor size, 3) the tumor grade and the histological type, 4) prior irradiation, 5) the presence of NF-1, and 6) the surgical margin status ${ }^{1,9,10,17,19)}$. However, the importance attributed to each of these factors has varied among different studies. Most reports agreed that a negative surgical margin is the most significant prognostic factor for survival and local control of disease $e^{1,2,6-8,10,13,15,17,19)}$. Therefore, a complete surgical excision is mandatory to achieve optimal local control and overall survival.

\section{CONCLUSION}

We report very rare case of primary cervical MPNST, which was removed through the occipital triangle. MPNSTs remain a diagnostic and a therapeutic challenge in many cases. Surgical resection is the mainstay of effective treatment and radiation therapy is helpful to reduce local recurrence. Because of the dismal prognosis, patients should be followed carefully to identify local recurrence or metastasis as early as possible.

\section{References}

1. Anghileri M, Miceli R, Fiore M, Mariani L, Ferrari A, Mussi C, et al : Malignant peripheral nerve sheath tumors : prognostic factors and survival in a series of patients treated at a single institution. Cancer $107: 1065-1074,2006$

2. Baehring JM, Betensky RA, Batchelor TT : Malignant peripheral nerve sheath tumor : the clinical spectrum and outcome of treatment. Neurology $61:$ 696-698, 2003

3. Bhattacharyya AK, Perrin R, Guha A : Peripheral nerve tumors : management strategies and molecular insights. J Neurooncol 69: 335-349, 2004

4. Cardona S, Schwarzbach M, Hinz U, Dimitrakopoulou-Strauss A, Attigah N, Mechtersheimer section sign G, et al : Evaluation of F18deoxyglucose positron emission tomography (FDG-PET) to assess the nature of neurogenic tumours. Eur J Surg Oncol 29 : 536-541, 2003

5. Carli M, Ferrari A, Mattke A, Zanetti I, Casanova M, Bisogno G, et al : Pediatric malignant peripheral nerve sheath tumor : The Italian and German soft tissue sarcoma cooperative group. J Clin Oncol $23: 8422-8430,2005$

6. Chandler CL, Uttley D, Wilkins PR, Kavanagh TG : Primary spinal malignant schwannoma. Br J Neurosurg 8 : 341-345, 1994

7. Doorn PF, Molenaar WM, Butler J, Hoekstra HJ : Malignant peripheral nerve sheath tumors in patients with and without neurofibromatosis. Eur J Surg Oncol 21 : 78-82, 1995

8. Ducatman BS, Scheithauer BW, Piepgras DG, Reiman HM, Ilstrup DM : Malignant peripheral nerve sheath tumors. A clinicopathologic study of 120 cases. Cancer $57: 2006-2021,1986$

9. Kim JK, Song JW, Park WM, Jang JS, Rhee CH, Lee SH : Three cases of primary spinal malignant schwannomas. J Korean Neurosurg 
Soc $28: 720-725,1999$

10. Koea JB, Leung D, Lewis JJ, Brennan MF : Histopathologic type : an independent prognostic factor in primary soft tissue sarcoma of the extremity? Ann Surg Oncol 10 : 432-440, 2003

11. Louis DN, Ohgaki H, Wiestler OD, Cavenee WK (Eds.) : WHO classification of tumours of the central nervous system, ed 4. IARC : Lyon, 2007, pp160-162

12. Murphey MD, Smith WS, Smith SE, Kransdorf MJ, Temple HT : Imaging of musculoskeletal neurogenic tumors : radiologicpathologic correlation. RadioGraphics 19: 1253-1280, 1999

13. Perrin RG, Guha A : Malignant peripheral nerve sheath tumors. Neurosurg Clin N Am 15 : 203-216, 2004

14. Peter AH, Hubert RF, Suresh M, Paula C : Malignant peripheral nerve sheath tumor. RadioGraphics $23:$ 790-794, 2003
15. Stephen RG, John DR, Amir S, Charles HB, Steven NH : Malignant peripheral nerve sheath tumor. J Surg Oncol $97: 340-349,2008$

16. Vauthey JN, Woodruff JM, Brennan MF : Extremity malignant peripheral nerve sheath tumors (neurogenic sarcomas) : A 10-year experience. Ann Surg Oncol 2 : 126-131, 1995

17. Wanebo JE, Malik JM, VandenBerg SR, Wanebo HJ, Driesen N, Persing JA : Malignant peripheral nerve sheath tumors. A clinicopathologic study of 28 cases. Cancer $71: 1247-1253,1993$

18. Weiss SW, Langloss JM, Enzinger FM : Value of S-100 protein in the diagnosis of soft tissue tumors with particular reference to benign and malignant Schwann cell tumor. Lab Invest 49 : 299-308, 1983

19. Wong WW, Hirose T, Scheithauer BW, Schild SE, Gunderson LL : Malignant peripheral nerve sheath tumor : analysis of treatment outcome. Int J Radiat Oncol Biol Phys 42 : 351-360, 1998 